\title{
RANKL-binding peptide promotes ectopic bone formation induced by BMP-2 gene transfer in murine gastrocnemius muscle
}

\author{
Shigeki Nagahiro ${ }^{1}$, Tomoki Uehara ${ }^{1}$, Mariko Yamamoto Kawai ${ }^{2}$, Preksa Keo ${ }^{3}$, Toshimi Sato ${ }^{4}$, Hiroki Ochi ${ }^{5}$, Shingo Sato ${ }^{6}$, Shinji Kuroda ${ }^{7}$, \\ Takashi Ono ${ }^{3}$, Michiyo Miyashin ${ }^{1}$ and Kazuhiro Aoki ${ }^{8 *}$ \\ ${ }^{1}$ Department of Pediatric Dentistry / Dentistry for Persons with Special Needs, Graduate School of Medical and Dental Sciences, Tokyo Medical and Dental \\ University, Tokyo, Japan \\ ${ }^{2}$ Department of Medical Secretarial Arts, Kansai Women’s College, Osaka, Japan \\ ${ }^{3}$ Department of Orthodontic Science, Graduate School of Medical and Dental Sciences, Tokyo Medical and Dental University, Tokyo, Japan \\ ${ }^{4}$ Department of Bio-Matrix (Pharmacology), Graduate School of Medical and Dental Sciences, Tokyo Medical and Dental University, Tokyo, Japan \\ ${ }^{5}$ Department of Orthopaedic and Trauma Research, Graduate School of Medical and Dental Sciences, Tokyo Medical and Dental University, Tokyo, Japan \\ ${ }^{6}$ Center for Innovative Cancer Treatment, Tokyo Medical and Dental University Hospital, Tokyo, Japan \\ ${ }^{7}$ Department of Oral Implantology and Regenerative Dental Medicine, Graduate School of Medical and Dental Sciences, Tokyo Medical and Dental University, \\ Tokyo, Japan \\ ${ }^{8}$ Department of Basic Oral Health Engineering, Graduate School of Medical and Dental Sciences, Tokyo Medical and Dental University, Tokyo, Japan
}

\begin{abstract}
Bone morphogenetic protein-2 (BMP-2) gene delivery is expected to be useful as a way to overcome protein delivery problems, such as the need for a scaffold and high dose of BMP-2 protein, and using non-viral vectors is known to be a safer gene delivery system than that using viral vectors for clinical application. However, the efficiency of the BMP-2 expression is very low when non-viral vectors are used. In the present study, we clarified the feasibility of RANKL-binding peptide, which has recently been recognized as a bone anabolic reagent, in bone formation induced by BMP-2 gene transfer using non-viral vectors. We induced ectopic bone in the gastrocnemius muscle of C57BL/6 mice by a single injection of GFP/BMP-2-expressing vector. Subcutaneous administration of RANKL-binding peptide increased the ectopic bone mass on days 14 and 21 after plasmid injection, and histological analyses showed the stimulation of bone formation activity of the ectopic bone compared to the vehicle-treated control. Micro computed tomography images showed that RANKL-binding peptide improved the microstructure of ectopic bone. Our results suggest that RANKL-binding peptide may be effective in promoting bone formation induced by BMP- 2 gene transfer using non-viral vectors.
\end{abstract}

\section{Introduction}

Bone morphogenetic protein-2 (BMP-2), a bone-formation inducer, has been widely used in bone regeneration [1]. However, there are several critical problems associated with its clinical application, such as the need for a scaffold material and a high dose to induce bone formation [2,3]. BMP-2 gene delivery is believed to be one way of overcoming these problems with protein delivery $[1,2,4]$. The direct injection of genetic materials, such as via a plasmid vector, can be performed to induce bone formation without using a scaffold material, since gene-transfected cells can release the protein similarly to the scaffold materials, releasing the protein sustainably over a given duration [2]. Gene transfer is therefore considered a promising approach to BMP-2 therapy.

Various routes for BMP-2 gene transfer have been developed, with two kinds of vectors mainly used: non-viral and viral vectors [1]. Since viral vectors have a higher gene transfer efficiency than non-viral vectors, bone induction is greater when the BMP-2 gene is transfected using a viral vector $[5,6]$. Indeed, when we used a non-vial vector of BMP-2 to induce bone through electroporation, very little ectopic bone was generated in rat skeletal muscle [7,8]. Even after increasing the amount of plasmid used or the number of rounds of electroporation, the amount of bone induced was relatively unchanged [7]. Despite the development of a number of other methods for increasing the amount of bone induced using non-viral vectors, such as sonoporation (cell sonication) [9] and cationic polymers [1,10], the amount of bone induced remains insufficient for clinical applications.

The receptor activator of NF- $\kappa B$ ligand (RANKL)-binding peptide is known to accelerate BMP-2-induced bone formation both in vitro

${ }^{\star}$ Correspondence to: Kazuhiro Aoki, Professor, Department of Basic Oral Health Engineering, Graduate School of Medical and Dental Sciences, Tokyo Medical and Dental University, 1-5-45, Yushima, Bunkyo-ku, Tokyo, 113-8549, Japan, Tel: +813-5803-4641, E-mail: kazu.hpha@tmd.ac.jp

Key words: BMP-2, gene transfer, non-viral vector, RANKL-binding peptide, ectopic bone

Received: December 08, 2019; Accepted: December 30, 2019; Published: January 07, 2020 
and in vivo [11-14]. RANKL-binding peptides are thought to bind to the cellular surface acceptor RANKL on osteoblasts, leading to the expression of Runx-2, a master gene of osteoblast differentiation $[15,16]$. While this strategy combining RANKL-binding peptide and BMP2 in protein form has been shown to stimulate BMP-2-induced bone formation in many animal models [12-14,17], the stimulatory effects of RANKL-binding peptide on bone formation induced by BMP-2 gene transfer have not been investigated. Since the amount of bone induced by BMP-2 gene transfer was slight even when using electroporation as described above, we hypothesized that RANKL-binding peptide might stimulate the bone formation induced by a non-viral vector.

The present study therefore assessed the feasibility of RANKLbinding peptide in bone formation induced by BMP-2 gene transfer through electroporation using a non-viral vector.

\section{Materials and methods}

\section{Plasmid DNA of BMP-2 and a RANKL-binding peptide}

pEGFP-N1 plasmid vector (Clontech, Mountain View, CA, USA) harboring CDNA of human BMP-2 in the BamHI restriction enzyme region at the multicloning site was used for BMP-2 gene transfer [18]. WP9QY (W9; YCWSQYLCY), a RANKL-binding peptide [19], was kindly provided by JITSUBO Co., Ltd. (Kanagawa, Japan).

\section{Cell culture and gene transfer in vitro}

ST2 cells (a murine osteoblastic cell line; RIKEN, Ibaragi, Japan) were seeded at $1 \times 10^{4} /$ well in a 48 -well plate and cultured in growth medium (DMEM) supplemented with $10 \%$ fetal bovine serum (FBS; GE Healthcare Japan, Tokyo, Japan) 1 day before lipofection. The next day, $0.2 \mu \mathrm{g} / \mu \mathrm{l}$ of EGFP-hBMP-2 vector was diluted in Opti-MEM (Invitrogen, Carlsbad, CA, USA) and then mixed with Lipofectamine 2000 (Invitrogen) for lipofection. Twelve hours after lipofection, the medium was changed. One day after lipofection, the culture was stimulated with $100 \mu \mathrm{M}$ of W9. To the wells without W9, only vehicle was added. Ten days after lipofection, the cells were rinsed with phosphate-buffered saline (PBS), harvested, and lysed with lysis buffer (0.1\% Triton-X; Nacalai Tesque Inc., Kyoto, Japan) including $10 \mathrm{mM}$ Tris-HCL. The protein concentration in the lysis buffer was determined using a BCA Protein Assay Kit (Takara Bio Inc., Shiga, Japan), and the alkaline phosphatase (ALP) activity was measured using a Lab Assay ALP (Wako Pure Chemical Corp., Osaka, Japan) following the manufacturer's protocol and a microplate reader (iMark; Bio-Rad, Hercules, CA, USA).

\section{BMP-2 gene transfer in the gastrocnemius muscle in vivo}

Eight-week-old male C57BL/6 mice were obtained from Nippon CLEA (Tokyo, Japan), and maintained as described elsewhere [20]. All animal experiments were approved by the Institutional Animal Care and Use Committee of Tokyo Medical and Dental University (Tokyo, Japan; authorization numbers: A2018-104C, A2019-155A). Before treatment, mice were anesthetized as previously described [21]. We performed BMP-2 gene transfer into mouse gastrocnemius muscle using the plasmid vector expressing BMP-2 via electroporation, as previously described [7,22,23] (Figure 1A).

\section{Quantification of GFP-BMP-2 fusion protein expressions in the gastrocnemius muscle}

Eighteen mice were divided into 6 groups $(n=3)$ to clarify the amount of protein expression induced by in vivo gene transfer of the GFP-BMP-2 expression vector. Gene transfer to the gastrocnemius muscle was performed as described above. Empty vector without the BMP-2 gene (pCAGGS) was injected as a control vector (mock). Mice were sacrificed under anesthesia by cervical dislocation on days 1,7 , or 14 after gene transfer (Figure 1B), and the gastrocnemius muscle was dissected. GFP fluorescence images at the gene transfer site were obtained by fluorescent microscopy (FSX100; Olympus, Tokyo, Japan) using the undecalcified sections prepared as previously described [13]. The dissected gastrocnemius muscle $(0.15 \mathrm{~g})$ was homogenized with $0.25 \mathrm{M}$ sucrose by a Polytron homogenizer (type ABM; Nissei Inc., Osaka, Japan) and then centrifuged at 5,000 $\mathrm{g}$ for $10 \mathrm{~min}$ at $4{ }^{\circ} \mathrm{C}$. The protein concentration in the supernatant was measured as described above. The GFP expression in the supernatant was measured using a GFP-specific ELISA kit (Cell Biolabs, Inc., San Diego, CA, USA), and the relative GFP protein expression (pg) against the total protein amount $(\mu \mathrm{g})$ was calculated. The total amount of BMP-2 was calculated from the GFP amounts.

\section{Experimental design of ectopic bone formation induced by BMP-2 gene transfer using RANKL-binding peptide}

Ten mice were divided into 2 groups $(\mathrm{n}=5)$, and in vivo gene transfer to the gastrocnemius muscle was performed as described above. After gene transfer, RANKL-binding peptide (W9; $10 \mathrm{mg} / \mathrm{kg} /$ day) or vehicle was subcutaneously administered using osmotic minipumps (Model 2001; Alzet, Palo Alto, CA, USA) to the BMP-2+W9 and BMP$2+\mathrm{VEH}$ groups, respectively [24]. Alizarin complexone $(20 \mathrm{mg} / \mathrm{kg}$; Dojindo, Kumamoto, Japan) was injected on day 12 to measure the bone formation activity. The mice were sacrificed under anesthesia by cervical dislocation on day 21 (Figure 1C).

\section{Radiological assessments}

Soft X-ray images were taken with a cabinet X-ray device (type SRO-M50; Sofron, Tokyo, Japan). In vivo microfocal computed tomography (R_mCT2; Rigaku, Tokyo, Japan) was performed at

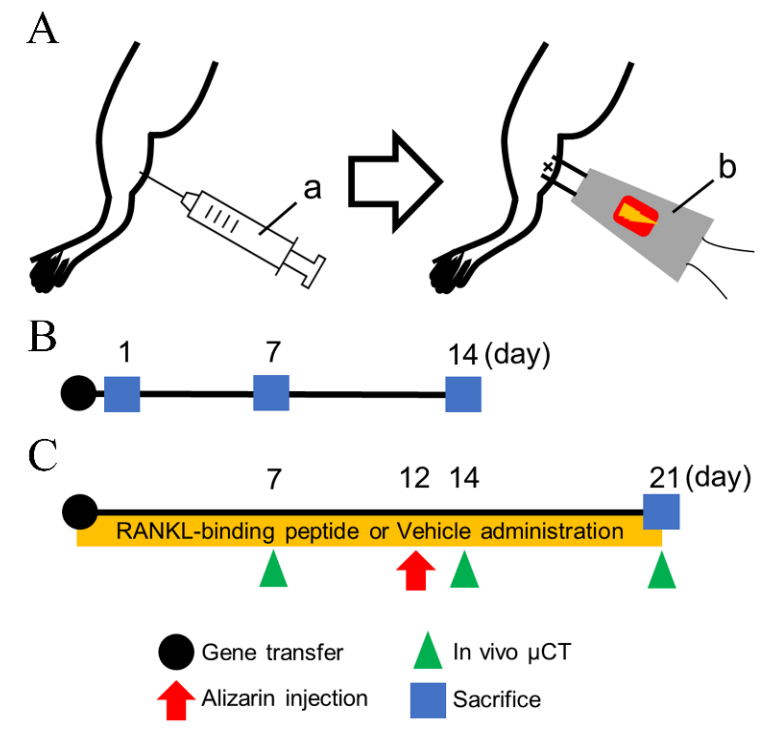

Figure 1. Experimental design. A. A schematic view of the gene transfer. Electroporation $(100 \mathrm{~V}, 50 \mathrm{~ms}, 8$ pulse) was carried out immediately after the injection of BMP-2expressing plasmid vector $(0.5 \mu \mathrm{g} / \mu \mathrm{L}$, total volume of $25 \mu \mathrm{L})$. (a) A 30-G syringe for plasmid injection. (b) Electroporator with needle-type electrodes. B. The GFP-BMP-2 expression was evaluated on days 1, 7, and 14. C. Osmotic minipumps filled with RANKLbinding peptide W9 or vehicle were subcutaneously implanted after gene transfer. In vivo $\mu \mathrm{CT}$ was performed weekly. Alizarin complexone for measuring the osteogenic activity was injected on day 12. Mice were sacrificed on day 21 
$90 \mathrm{kV}, 160 \mu \mathrm{A}$, and FOV 20 to obtain 3-dimensional bone images of each mouse every week until day 21 . Micro-architectural changes in ectopic bone were measured using an image analysis software program (TRI/3D-BON; RATOC System Engineering, Tokyo, Japan) [25]. The reduction rate (\%) was calculated as the ratio of the bone volume (BV) or bone surface (BS) on day 21 to that at day 14: [(BV or BS on day $14-$ $\mathrm{BV}$ or BS on day 21$) /(\mathrm{BV}$ or BS on day 14$)] \times 100$.

\section{Histological assessments and bone histomorphometry}

We prepared undecalcified sections as previously described [13]. Some sections were stained with tartrate-resistant acid phosphatase (TRAP) or von Kossa for histological assessments. Bone resorption parameters (N.Oc/BS, Oc.S/BS) and the calcified tissue thickness in the ectopic bone were measured according to standard bone histomorphometric analyses [26]. Alizarin-labeled images were used to evaluate the bone formation activity in the ectopic bone. The same threshold value of fluorescent intensity on day 12 was used to analyze the alizarin-positive area in all samples, and the image was binarized for the area measurement using the ImageJ software program (version 1.50i; NIH, Bethesda, MD, USA).

\section{Statistical analyses}

All data are presented as the means \pm standard deviation (SD). The statistical significance between two groups was compared using an unpaired $t$-test. Multiple groups comparisons were performed using a one-way analysis of variance and Tukey's multi-comparison post hoc test. P values of $<0.05$ were considered to be significant. We also assumed that $0.05<\mathrm{P}<0.1$ indicated edge cases of significance or were highly suggestive of significance, according to the statement by the American Statistical Association [27,28].

\section{Results}

\section{Effects of RANKL-binding peptide W9 on osteoblast differentiation in BMP-2-gene-transfected ST2 cells}

To investigate whether or not W9 exerts a synergistic effect on osteoblast differentiation in BMP-2-gene transfected cells in vitro, we first added $100 \mu \mathrm{M}$ of W9 to ST2-cell culture. As shown in Figure $2 \mathrm{~A}, 100 \mu \mathrm{M}$ of W9 stimulated the activity of ALP, a marker of early osteoblast differentiation, without BMP-2 gene transfection. Although no significant increase in ALP activity was noted in the BMP-2-genetransfected wells compared to the vehicle control $(0 \mu \mathrm{M}$ W9), an increase was observed in the presence of $100 \mu \mathrm{M}$ of W9 in the BMP2-gene-transfected ST2 cells. Furthermore, it was also detected when compared to the culture with $100 \mu \mathrm{M}$ of W9 in ST2 cells without BMP-2 gene transfer.

\section{Chronological changes in GFP-BMP-2 fusion protein expression after gene transfer in gastrocnemius muscle}

We measured the GFP protein expression on days 1, 7, and 14 after gene transfer in the gastrocnemius muscle to evaluate the chronological changes in the GFP-BMP-2 fusion protein expression. As shown in Figure 2B, GFP-fluorescent-positive cells at the injection site of the plasmid vector were detected in the histological sections of the gastrocnemius muscle on day 1 after gene transfer, and these cells were increased on day 7 and were still observed on day 14 after gene transfer. The fluorescent images from the specimens after the transfection of the empty vector did not show any GFP-positive cells at any time points (Figure 2B). Quantitative analyses of the relative GFP expression confirmed these observations (Figure 2C). When we calculated the total amount of BMP-2 protein in the whole sample dissected from the gastrocnemius muscle, the average amount was 53,129 , and $100 \mathrm{pg}$ on days 1,7 , and 14, respectively (Table 1 ).

Effects of W9 on the microstructure of ectopic bone induced by BMP-2 gene transfer

To investigate the effects of W9 on the bone mass and microstructure of the ectopic bone induced by BMP-2 gene transfer, W9 or vehicle was subcutaneously administered to BMP-2-genetransfected mice. Radiological images showed a greater amount of ectopic bone in the BMP-2+W9 group than in the BMP-2+VEH group (Figures $3 \mathrm{~A}$ and $\mathrm{B}$ ), and the bone volume analyses, such as the $\mathrm{BMD}$ and $\mathrm{BV} / \mathrm{TV}$, of the ectopic bone confirmed these observations (Figures 3C and D). The SMI, an indicator of a plate-like structure, was significantly lower in the BMP-2+W9 group than in the BMP$2+\mathrm{VEH}$ group on day 14 , and all parameters related to the bone microstructure were significantly changed on day 21 , showing improvement in the bone microstructure (Figures 3E-3H). The reduction rate of the ectopic bone from day 14 to day 21 was slower in the BMP-2+W9 group than in the BMP-2+VEH group (Figures $3 \mathrm{I}$ and $3 \mathrm{~J})$.

Effects of W9 on osteoclast formation and bone formation activity of ectopic bone induced by BMP-2 gene transfer

To clarify the functional role of W9 on BMP-2-gene-transferinduced bone, histological analyses were performed. As shown in
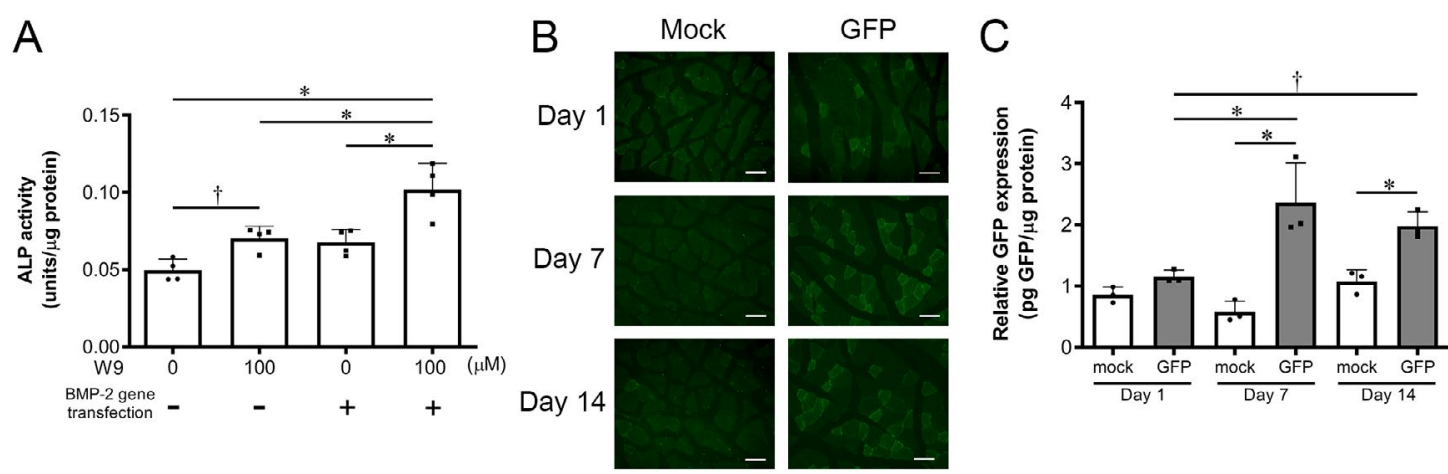

Figure 2. W9 promoted osteoblast differentiation in ST2 cells in vitro and chronological changes in the GFP-BMP-2 fusion protein expression in vivo. A. ALP activity in ST2 cells with or without transfection of a BMP-2 gene-expressing plasmid vector in the presence or absence of $100 \mu \mathrm{M}$ W9 on day 10 after gene transfection. Data are shown as the mean \pm SD. ${ }^{*} \mathrm{p}<0.05 .{ }^{\dagger} 0.05<\mathrm{p}<0.1$. B. Fluorescence images of GFP-positive muscle cells on days 1,7 , and 14 after GFP-BMP2 gene transfer (GFP) or empty vector transfer (mock). Scale bars $=0.05$ mm. C. Relative GFP expression on days 1,7 , and 14 after gene transfer. Data are shown as the mean \pm SD. ${ }^{*} \mathrm{p}<0.05 .{ }^{\dagger} 0.05<\mathrm{p}<0.1$ 
Table 1. The amount of protein in the GFP-BMP-2-gene transfected gastrocnemius muscle

\begin{tabular}{|c|c|c|c|}
\hline & Day 1 & Day 7 & Day 14 \\
\hline GFP $(\mathrm{pg})$ & $55.1 \pm 6.9$ & $134.0 \pm 36.4$ & $104.8 \pm 17.1$ \\
\hline BMP-2 $(\mathrm{pg})$ & $53.1 \pm 6.7$ & $129.1 \pm 35.0$ & $100.9 \pm 16.5$ \\
\hline Total protein amount $(\mu \mathrm{g})$ & $47.5 \pm 2.4$ & $56.1 \pm 3.6$ & $52.5 \pm 3.8$ \\
\hline
\end{tabular}

The GFP concentration in the homogenized tissue solution from the dissected muscle, which was transfected by plasmid vector including the GFP-BMP-2 gene, were measured using an ELISA kit. Then, the GFP amount in the dissected muscle was calculated from the GFP concentration measured by ELISA. The total amount of BMP-2 was calculated from the GFP amounts. The total protein amount in the dissected muscle was measured using a standard protocol. The details have been described in the Materials and Method section. Data are shown as the mean $\pm \operatorname{SD}(n=3)$

A
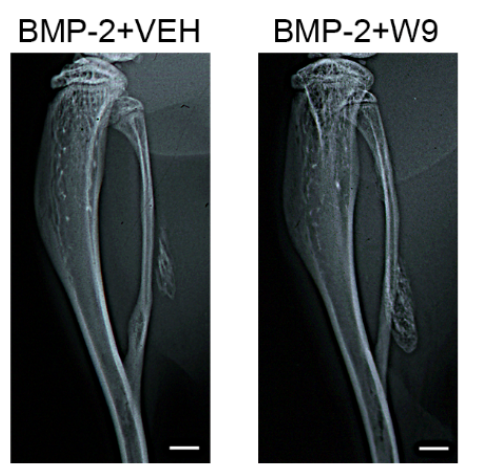

B

BMP-2+VEH
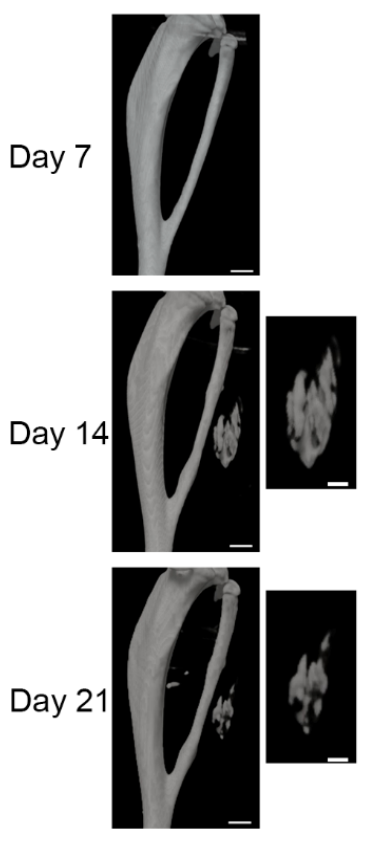

BMP-2+W9
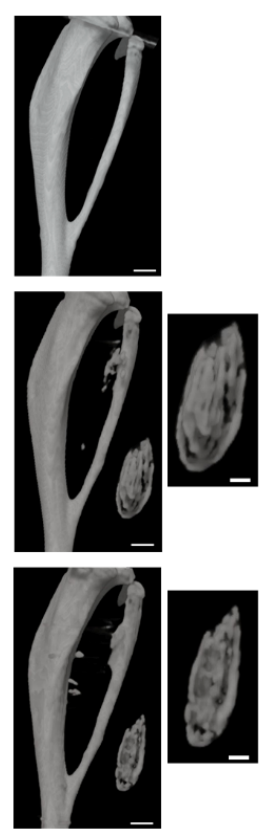

C

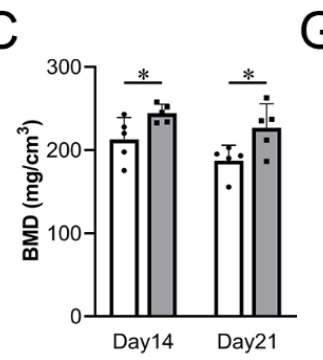

G

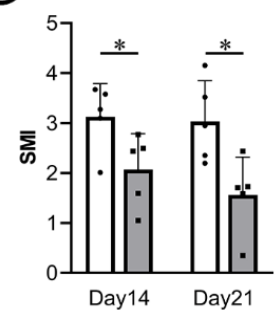

$\mathrm{D}$

H
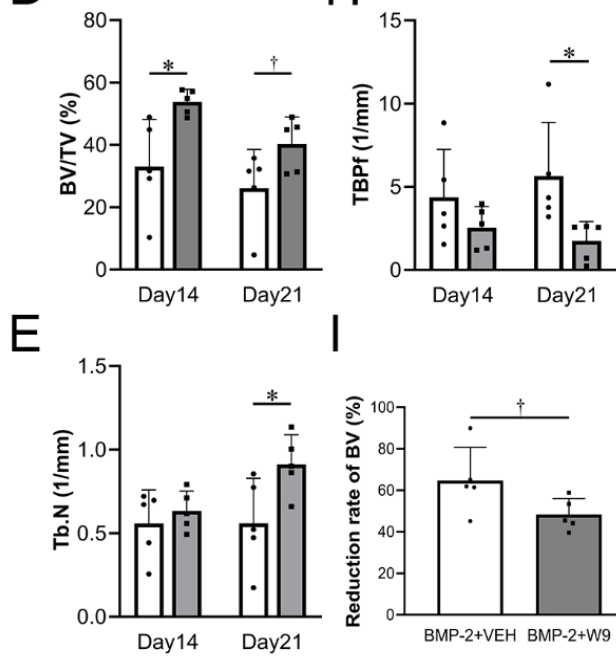

$\mathrm{F}$
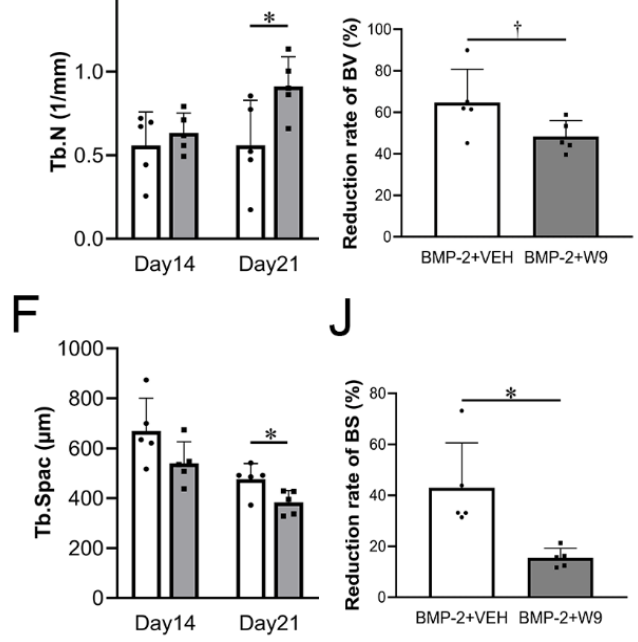

$\mathrm{J}$

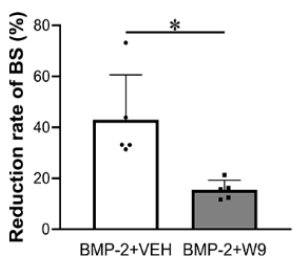

Figure 3. Radiological analyses revealed increased ectopic bone formation and structurally improved bone induced by BMP-2-gene transfer in the W9-treated group. A. Soft X-ray photographic images. BMP-2+VEH: BMP-2 gene transfer + vehicle-treated group, BMP-2+W9: BMP-2 gene transfer + W9-treated group. Scale bars=1 mm. B. $\mu$ CT images of lower limb and ectopic bone on days 7, 14, and 21. Scale bars=1 mm (low magnification), $0.5 \mathrm{~mm}$ (high magnification). C. Bone mineral density (BMD) D. Ratio of bone volume (BV) to tissue volume (TV) E. Trabecular number (Tb.N) F. Trabecular spacing (Tb.Spac) G. Structure model index (SMI) H. Trabecular bone pattern factor (TBPf) I and J. The reduction rate calculated from the $\mathrm{BV}$ and BS on days 14 and 21 . White bar: BMP-2+VEH, Gray bar: BMP-2+W9. Data are shown as the mean \pm SD. ${ }^{*} \mathrm{p}<0.05$ vs. Ctr. ${ }^{\dagger} 0.05<\mathrm{p}<0.1$ vs. Ctr

Figure 4A, TRAP-positive cells were less frequent in the BMP-2+W9 group than in the BMP-2+VEH group. The alizarin-labeled area, which indicated the active bone formation area, seemed to be larger in the BMP-2+W9 group than in the BMP-2+VEH group. Quantitative analyses confirmed these observations (Figures $4 \mathrm{~B}$ and $\mathrm{E}$ ). The calcified tissue thickness also confirmed radiological observations of ectopic bone (Figures 3B and 4D).

\section{Discussion}

The amount of BMP-2 protein needed to induce bone formation is far beyond the physiological amount that is available [2]. In our previous study, $1 \times 10^{6} \mathrm{pg}$ of BMP- 2 was deemed necessary to form bone [29], and $3 \times 10^{5} \mathrm{pg}$ of BMP-2 was unable to form bone efficiently in a bone defect model [14]. In the present study, the amount of BMP-2 


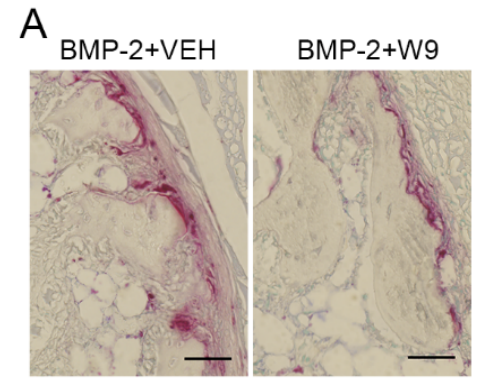

B

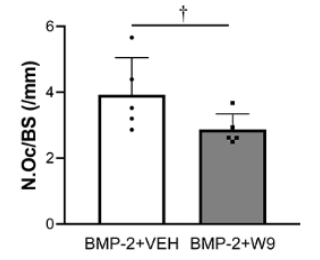

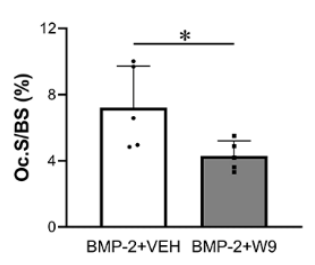

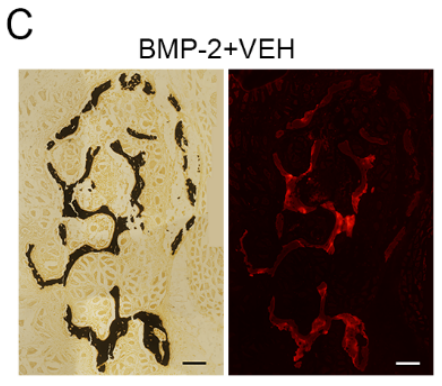

$\mathrm{D}$

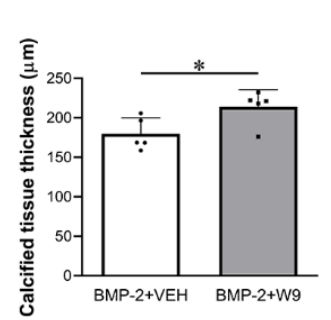

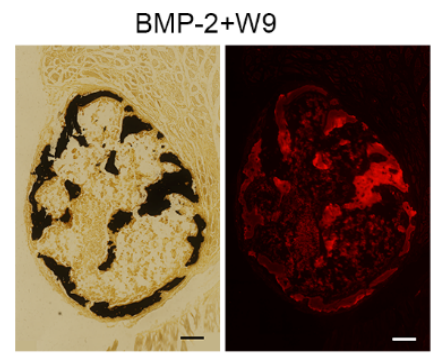

E

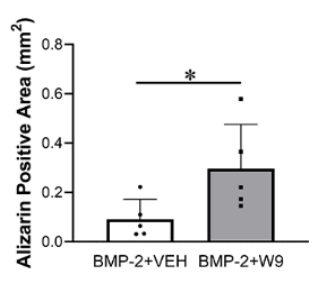

Figure 4. W9 inhibited osteoclast formation and accelerated mineralization of ectopic bone induced by BMP-2 gene transfer. A. TRAP-stained images of ectopic bone. TRAP-positive cells are shown in red. Scale bars=0.05 mm. B. Number of osteoclast/bone surface (N.Oc/BS), osteoclast surface/bone surface (Oc.S/BS) C. Bright field images of von-Kossa-stained undecalcified sections of ectopic bone (calcified tissue is shown in black). Fluorescent images of the alizarin-positive area (shown in red). Scale bars=0.1 mm. D. Calcified tissue thickness calculated based on the von-Kossa-positive area and perimeter. E. A quantitative analysis of the alizarin-positive area. Data are shown as the mean $\pm \mathrm{SD}$. ${ }^{\mathrm{p}}<0.05 \mathrm{vs.} \mathrm{Ctr.} \dagger 0.05<\mathrm{p}<0.1$ vs. Ctr. See Figure 3A for other definitions

protein expressed in the dissected gastrocnemius muscle at the BMP2 gene transfer site was less than $200 \mathrm{pg}$ at all time points after gene transfer during the experiments (Table 1). Since the amount of protein in the bone defect model was the total amount of BMP-2 incorporated in the scaffold material available for 28 days, while it was measured in the dissected gastrocnemius muscle at each time point in the present study, it might not be possible to compare these two studies. However, our findings suggest at least that very little BMP-2 was required to induce bone formation, although the amount formed was very small (Figure 3).

Since a high level of BMP-2 has been recognized as a cause of inflammation and/or carcinogenicity [30], many studies have attempted to reduce the amount of BMP-2 needed to induce bone formation. The BMP-2 expression in our study was very low, as shown in Table 1 , suggesting that gene therapy using a BMP-2 plasmid vector may be a promising therapy; however, the actual bone formation induced by BMP-2 gene transfer alone was consequently quite little. In the present study, we used RANKL-binding peptide to increase the bone formation induced by BMP-2 gene transfer through electroporation, and this approach actually significantly enhanced the BV/TV and alizarinpositive area compared to the vehicle control group (Figures $3 \mathrm{D}$ and $4 \mathrm{E}$ ), suggesting that RANKL-binding peptide stimulated BMP-2-genetransfer-induced bone formation. We also found that RANKL-binding peptide increased the bone quality, as indicated by improvement in microstructural parameters such as the SMI and TBPf (Figures 3E$3 \mathrm{H})$ [25]. The present study revealed the beneficial effects of RANKLbinding peptide on bone formation and the quality of bone induced by BMP-2 gene transfer.

Ectopic bone formation was not detected on day 7 and only first appeared from day 14 after gene transfer in both the vehicle and RANKL-binding peptide-treated groups (Figure $3 \mathrm{~B}$ ). The BV/TV was increased on day 14 (Figure 3D), and the alizarin-positive area, which was the indicator of bone formation activity, was increased on day 12 after gene transfer in the RANKL-binding peptide group compared to the vehicle control group (Figure 4E). These results suggest that the increase in the bone formation activity on day 12 may lead to an increase in the ectopic bone volume induced by BMP-2 gene transfer.

\section{Conclusion}

Our data suggest that RANKL-binding peptide was effective in promoting bone formation induced by BMP-2 gene transfer through electroporation using a non-viral vector. Therefore, the combination of the use of RANKL-binding peptide and the BMP-2 gene transfer might be an innovative method for use in bone regenerative therapy.

\section{Acknowledgements}

The authors thank Drs. Yukihiko Tamura, Hitoyata Shimokawa, (Graduate School of Medical and Dental Sciences, Tokyo Medical and Dental University, Tokyo, Japan) for their support of this experiment.

\section{Funding}

This study was supported by JSPS KAKENHI to TU (17K17314) and KA (17K11900).

\section{Conflicts of interest}

The authors declare no conflicts of interest.

\section{References}

1. Park SY, Kim KH, Kim S, Lee YM, Seol YJ (2019) BMP-2 gene delivery-based bone regeneration in dentistry. Pharmaceutics 11(8).

2. Fischer J, Kolk A, Wolfart S, Pautke C, Warnke PH, et al. (2011) Future of local bone regeneration - Protein versus gene therapy. J Craniomaxillofac Surg 39(1): 54-64.

3. Sheikh Z, Javaid MA, Hamdan N, Hashmi R (2015) Bone regeneration using bone morphogenetic proteins and various biomaterial carriers. Materials (Basel) 8(4): 17781816.

4. Im GI (2013) Nonviral gene transfer strategies to promote bone regeneration. J Biomed Mater Res A 101(10): 3009-18.

5. Sugiyama O, An DS, Kung SP, Feeley BT, Gamradt S, et al. (2005) Lentivirus-mediated gene transfer induces long-term transgene expression of BMP-2 in vitro and new bone formation in vivo. Mol Ther 11(3): 390-398. 
6. Abe N, Lee YP, Sato M, Zhang X, Wu J, et al. (2002) Enhancement of bone repair with a helper-dependent adenoviral transfer of bone morphogenetic protein-2. Biochem Biophys Res Commun 297(3): 523-527.

7. Kawai M, Bessho K, Kaihara S, Sonobe J, Oda K, et al. (2003) Ectopic bone formation by human bone morphogenetic protein-2 gene transfer to skeletal muscle using transcutaneous electroporation. Hum Gene Ther 14(16): 1547-1556.

8. Kawai M, Maruyama H, Bessho K, Yamamoto H, Miyazaki J, et al. (2009) Simple strategy for bone regeneration with a BMP-2/7 gene expression cassette vector. Biochem Biophys Res Commun 390(3): 1012-1017.

9. Osawa K, Okubo Y, Nakao K, Koyama N, Bessho K (2009) Osteoinduction by microbubble-enhanced transcutaneous sonoporation of human bone morphogenetic protein-2. J Gene Med 11(7): 633-641.

10. Komatsu K, Shibata T, Shimada A, Ideno H, Nakashima K, et al. (2016) Cationized gelatin hydrogels mixed with plasmid DNA induce stronger and more sustained gene expression than atelocollagen at calvarial bone defects in vivo. Journal of Biomaterials Science-Polymer Edition 27(5): 419-430.

11. Furuya Y, Inagaki A, Khan M, Mori K, Penninger JM, et al. (2013) Stimulation of bone formation in cortical bone of mice treated with a receptor activator of nuclear factorkappaB ligand (RANKL)-binding peptide that possesses osteoclastogenesis inhibitory activity. $J$ Biol Chem 288(8): 5562-5571.

12. Masud Khan AA, Alles N, Soysa NS, Al Mamun MA, Nagano K, et al. (2013) The local administration of TNF- $\alpha$ and RANKL antagonist peptide promotes BMP-2-induced bone formation. Journal of Oral Biosciences 55(1): 47-54.

13. Uehara T, Mise-Omata S, Matsui M, Tabata Y, Murali R, et al. (2016) Delivery of RANKL-Binding Peptide OP3-4 Promotes BMP-2-induced maxillary bone regeneration. J Dent Res 95(6): 665-672.

14. Sugamori Y, Mise-Omata S, Maeda C, Aoki S, Tabata Y, et al. (2016) Peptide drugs accelerate BMP-2-induced calvarial bone regeneration and stimulate osteoblast differentiation through mTORC1 signaling. Bioessays 38(8): 717-725.

15. Sone E, Noshiro D, Ikebuchi Y, Nakagawa M, Khan M, et al. (2019) The induction of RANKL molecule clustering could stimulate early osteoblast differentiation. Biochemical and Biophysical Research Communications 509(2): 435-440.

16. Ikebuchi Y, Aoki S, Honma M, Hayashi M, Sugamori Y, et al. (2018). Coupling of bone resorption and formation by RANKL reverse signalling. Nature 561(7722): 195-200.

17. Arai Y, Aoki K, Shimizu Y, Tabata Y, Ono T, et al. (2016) Peptide-induced de novo bone formation after tooth extraction prevents alveolar bone loss in a murine tooth extraction model. Eur J Pharmacol 782: 89-97.
18. Oda M, Kuroda S, Kondo H, Kasugai S (2009) Hydroxyapatite fiber material with BMP-2 gene induces ectopic bone formation. J Biomed Mater Res B Appl Biomater 90(1): 101-109.

19. Aoki K, Saito H, Itzstein C, Ishiguro M, Shibata T, et al. (2006) A TNF receptor loop peptide mimic blocks RANK ligand-induced signaling, bone resorption, and bone loss. $J$ Clin Invest 116(6): 1525-1534.

20. Saito H, Kojima T, Takahashi M, Horne WC, Baron R, et al. (2007) A tumor necrosis factor receptor loop peptide mimic inhibits bone destruction to the same extent as anti-tumor necrosis factor monoclonal antibody in murine collagen-induced arthritis. Arthritis and Rheumatism 56(4): 1164-1174.

21. Haque Bhuyan MZ, Tamura Y, Sone E, Yoshinari Y, Maeda C, et al. (2017) The intraarticular injection of RANKL-binding peptides inhibits cartilage degeneration in a murine model of osteoarthritis. J Pharmacol Sci 134(2): 124-130.

22. Kawai M, Kataoka YH, Sonobe J, Yamamoto H, Inubushi M, et al. (2018) Non-surgical model for alveolar bone regeneration by bone morphogenetic protein-2/7 gene therapy J Periodontol 89(1): 85-92.

23. Yamamoto H, Kawai M, Shiotsu N, Watanabe M, Yoshida Y, et al (2012) BMP-2 gene transfer under various conditions with in vivo electroporation and bone induction. Journal of Oral and Maxillofacial Surgery, Medicine, and Pathology 24(1): 49-53.

24. Kato G, Shimizu Y, Arai Y, Suzuki N, Sugamori Y, et al. (2015) The inhibitory effects of a RANKL-binding peptide on articular and periarticular bone loss in a murine model of collagen-induced arthritis: a bone histomorphometric study. Arthritis Res Ther 17: 251.

25. Bouxsein ML, Boyd SK, Christiansen BA, Guldberg RE, Jepsen KJ, et al. (2010) Guidelines for assessment of bone microstructure in rodents using micro-computed tomography. Journal of Bone and Mineral Research 25(7): 1468-1486.

26. Dempster DW, Compston JE, Drezner MK, Glorieux FH, Kanis JA, et al. (2013) Standardized nomenclature, symbols, and units for bone histomorphometry: A 2012 update of the report of the ASBMR Histomorphometry Nomenclature Committee. $J$ Bone Miner Res 28(1): 2-17.

27. Yaddanapudi LN (2016) The American Statistical Association statement on P-values explained. J Anaesthesiol Clin Pharmacol 32(4): 421-423.

28. Baker M (2016) Statisticians issue warning over misuse of P values. Nature 531(7593): 151.

29. Al Mamun MA, Khan MAAM, Alles N, Matsui M, Tabata Y, et al. (2013) Gelatin hydrogel carrier with the W9-peptide elicits synergistic effects on BMP-2-induced bone regeneration. Journal of Oral Biosciences 55(4): 217-223.

30. James AW, LaChaud G, Shen J, Asatrian G, Nguyen V, et al. (2016) A review of the clinical side effects of bone morphogenetic protein-2. Tissue Eng Part B Rev 22(4): 284-297.

Copyright: (C2020 Nagahiro S. This is an open-access article distributed under the terms of the Creative Commons Attribution License, which permits unrestricted use, distribution, and reproduction in any medium, provided the original author and source are credited. 